\title{
Multi-modality imaging to determine the cellular heterogeneity of nasopharyngeal carcinoma components
}

\author{
Weidong Zhang ${ }^{1, *}$, Yanling Zhang ${ }^{2,}{ }^{*}$, Shi $\mathrm{Ke}^{3}$, Mingjian Lu ${ }^{1, *}$, Guang Yang ${ }^{1}$, Tao \\ Zhang1, Jianjun Han ${ }^{1}$, Zhenyin Liu ${ }^{1}$, Wei Wang ${ }^{3}$, Henry Ran ${ }^{3}$, Chaoxia Zou ${ }^{3,4}$, \\ Shaofan Hu${ }^{5}$, Guangtao Lei ${ }^{6}$, Chuanxing Li ${ }^{1}$, Fujun Zhang ${ }^{1}$ \\ ${ }^{1}$ State Key Laboratory of Oncology in South China, Department of Imaging and Interventional Radiology, Cancer Center, Sun \\ Yat-sen University, Guangzhou, Guangdong, 510060, P. R. China \\ ${ }^{2}$ School of Biotechnology, Southern Medical University, Tonghe, Guangzhou, Guangdong, 510515, P.R. China. \\ ${ }^{3}$ Department of Radiology, Baylor College of Medicine, One Baylor Plaza, Houston, Texas, 77030, USA \\ ${ }^{4}$ Department of Biochemistry and Molecular Biology, Harbin Medical University, Harbin, Heilongjiang, 150081, P. R. China \\ 5 Jiangxi Children's Hospital, Nanchang, Jiangxi, 330006, P.R. China \\ ${ }^{6}$ Department of Cardiology, Second Affiliated Hospital of Nanchang University, Nanchang, Jiangxi, 330006, P.R. China \\ * These authors contributed equally to this manuscript \\ Correspondence to: Fujun Zhang, email: zhangfj@sysucc.org.cn \\ Chuanxing Li, email: lichh@sysucc.org.cn \\ Keywords: CT, FDG, CXCR4, retinoid acid, MMP, molecular imaging, multi-agent imaging, multi-modality imaging, optical imaging, \\ PET. \\ Received: February 13,2014 Accepted: April 11,2014 Published: April 11, 2014
}

This is an open-access article distributed under the terms of the Creative Commons Attribution License, which permits unrestricted use, distribution, and reproduction in any medium, provided the original author and source are credited.

\section{ABSTRACT:}

Nasopharyngeal carcinoma (NPC) is an endemic public health problem in South and Southeast Asian countries. The disease components at the molecular level are unclear and need exploration for the development of future individualized molecular medicine. The purpose of this study was to test the feasibility of target-specific agents to detect different components of NPC. The binding capability of human NPC cell lines was determined by incubation with either agents that specifically target the metabolic status, host cytokines, and stroma. Mice bearing human NPC xenografts were injected with the same test agents plus a clinical molecular imaging agent (18 F-fluorodeoxyglucose) and computer tomography (CT) contrast agent. In vitro cell studies have demonstrated that target-specific agents bind to NPC cells with significantly higher signal intensities. Those agents not only bound to the cell membrane but also penetrated into the cytosol and cell nuclei. In vivo imaging demonstrated that the human NPC xenografts revealed high glucose uptake and a profound vasculature in the tumor. All agents were bound to the tumor regions with a high tumor-to-muscle ratio. Finally, all imaging data were validated by histopathological results. Multiple, target-specific agents determine the dynamic and heterogeneous components of NPC at the molecular level.

\section{INTRODUCTION}

Nasopharyngeal carcinoma (NPC) is an endemic public health problem in South and Southeast Asian countries [1] with an incidence rate as high as 26.9 per 100,000 persons $[2,3]$. Current diagnosis still relies on non-specific imaging modalities such as X-ray, magnetic resonance imaging (MRI), computed tomography (CT) or ${ }^{18} \mathrm{~F}$-fluorodeoxyglucose (FDG)/positron emission tomography (PET). Additionally, the therapeutic regimens are standard for all patients. Thus, both the diagnosis and treatment lack specificity to the tumor components based on individual differences. Therefore, most patients only obtain a correct diagnosis at an advanced disease stage $[4,5]$, and their treatment outcomes are inconsistent. Furthermore, both the diagnostic and therapeutic 
approaches listed above critically need improvements, considering the individual differences of each patient, different disease types, variable disease stages, and various disease components. In addition, because advanced disease requires a multidisciplinary team to establish an optimal treatment strategy, a correct combination based on each patient's need and disease status is necessary to provide better disease control [6]. Understanding of the disease at the molecular level will improve the accuracy of diagnosis, efficiency of treatment and development of new treatment regimens. A regimen that combines target-specific therapies and is individualized to fit the personal needs of each patient will yield better outcomes and reduce systematic toxicity. Therefore, exploration of molecular targets becomes critical in the field.

It is well known that cancer is an evolving, dynamic, and heterogeneous system; the difficulty with its diagnosis and treatment supports the use of simultaneous, multitarget therapies [7]. Cellular heterogeneity and the timing of expression are critical characteristics of oncogenic events and provide a framework to interpret pathological, diagnostic, and therapeutic observations of tumors [7]. Current molecular imaging technology provides a noninvasive approach to accurately study multiple disease components longitudinally. The combination of nuclear medicine, optical molecular imaging, X-ray, and CT allows simultaneous detection of tumor molecular events.

In the present study, we utilized human NPC cell lines and tumor xenografts in nude mice to determine multiple disease components at the cellular or tumor levels. We found that NPC shows a high metabolic activity, cytokine response and stroma modification. Our data demonstrate that a single tumor has multiple cellular components, including tumor xenografts derived from homogeneous cell populations with the same genetic background and at the same time. We hope this work provides a different view of the complexity of human cancer and supports the use of simultaneous, multi-target therapies for treating cancer.

\section{RESULTS}

\section{In vitro imaging}

Binding of the retinoid acid (RA) agent to the NPC cell lines have been demonstrated in the previous publication [16, 17]. A further detail analysis and comparison of signal intensity demonstrated that the signal intensity of RA agent is statistically higher than that of the free dye (Fig. 1) with p-value of less than 0.0001 .

NPC cell line CNE-2 binding to the CXCR4 agent is shown in Fig. 2. This cell line was strongly positive for CXCR4 (Fig. 2A) with weak, nonspecific binding to the reporter dye (Fig. 2C). The signal intensity plots showed that the origins of the signal at the single-cell level had higher signal intensity than that of the cytosol for CXCR4 (Fig. 2B). The signal was diffuse in the cells binding to the nonspecific dye (Fig. 2D). Statistical comparison showed that the signal intensity of CXCR4 was significantly higher than that of the free dye (Fig. 2E).

The NPC cell line 5-8F was studied in detail regarding the binding location of the matrix metalloproteinase (MMP) agent, and the results are shown in Fig. 3. The signal channel images showed the cell morphology (Fig. 3A), cell membrane (Fig. 3B), MMP (Fig. 3C) and cell nuclei (Fig. 3D). Merged morphology and MMP imaging showed that the MMP signal arose from inside the cells (Fig. 3E), a finding that was confirmed by overlaying the cell membrane and MMP images (Fig. 3F). Merged cell nuclei and MMP images demonstrated that the MMP agent also penetrated the cell nuclear membrane (Fig. 3G). Finally, the location and relation of each component are shown in Fig. $3 \mathrm{H}$.

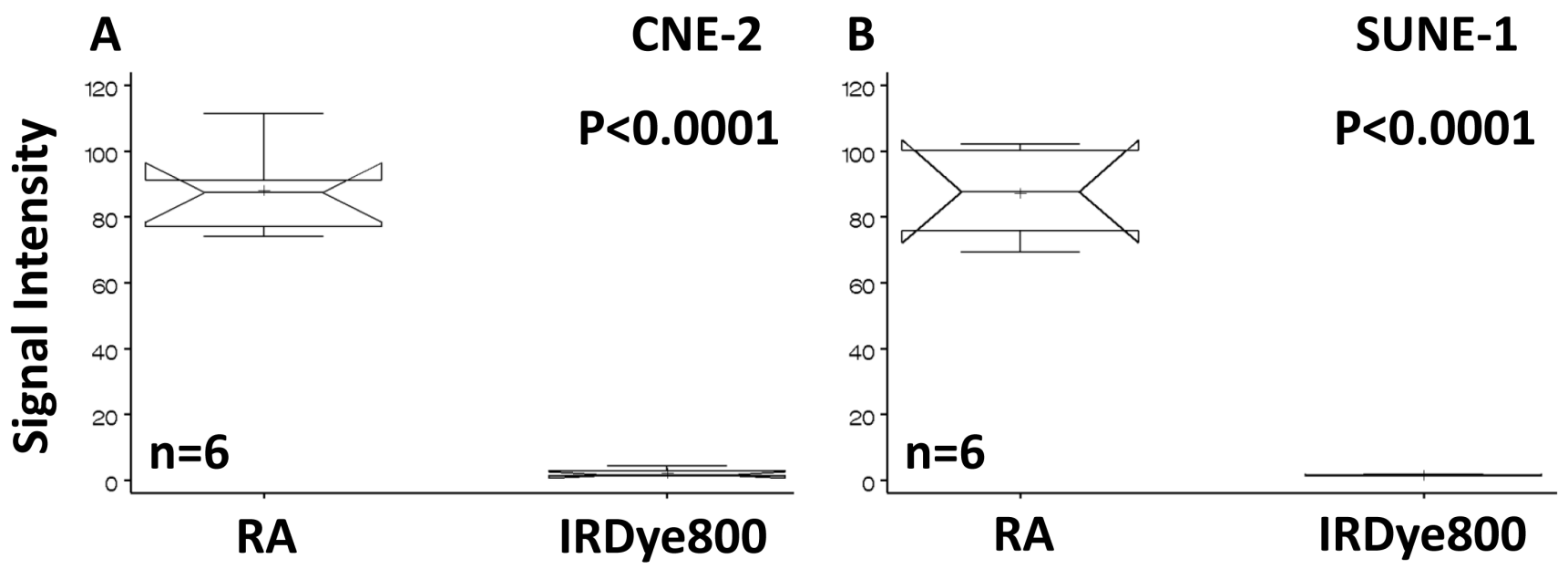

Fig 1: Statistical analysis and comparison of signal intensity of cells binding to RA. Human NPC cells CNE-2 (A) and SUNE1 (B) bound to the RA agent but not to the free reporter. The signal intensities of the RA agent are significantly higher than those of the dye. 

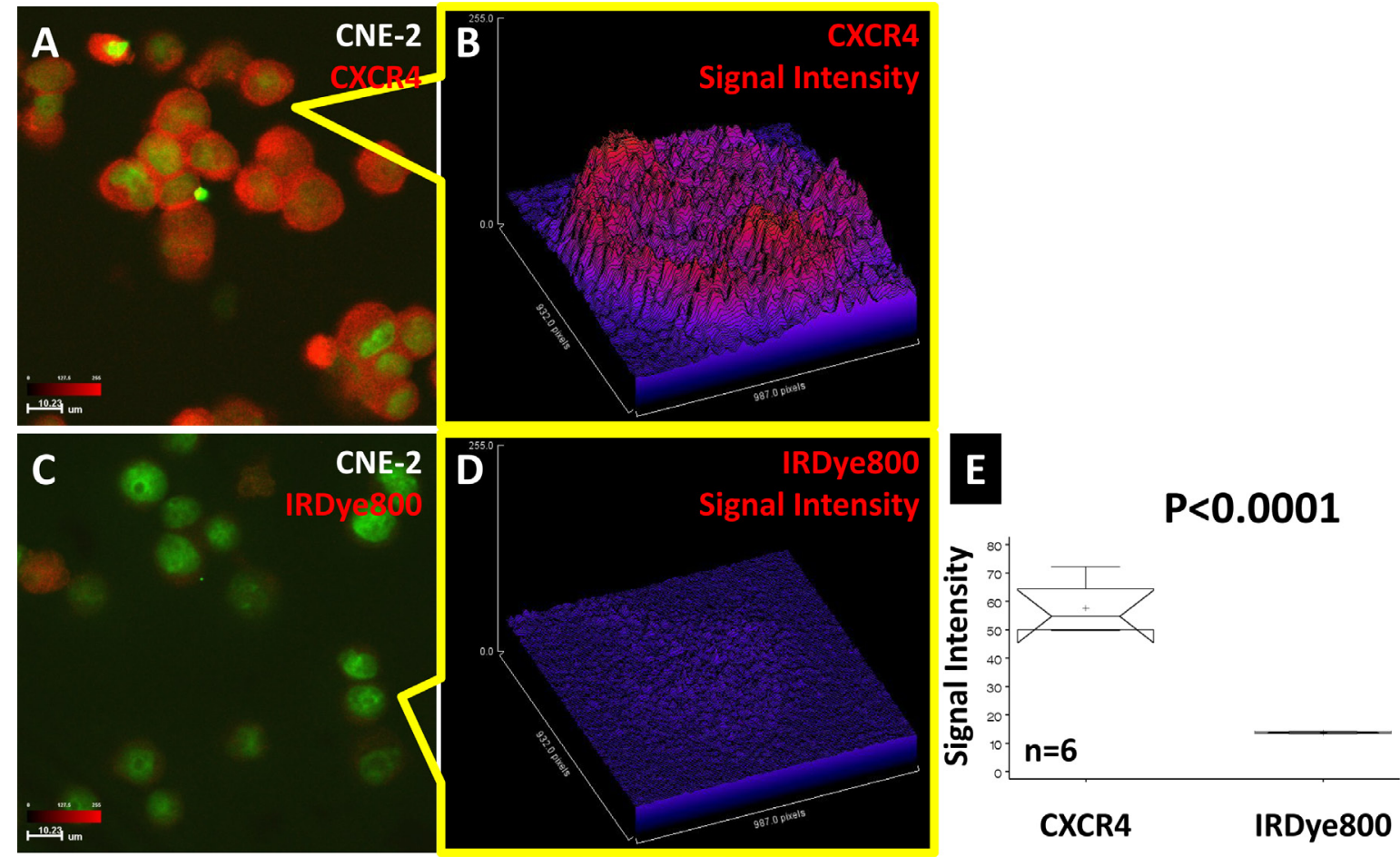

Fig 2: CXCR4 confocal imaging of human NPC CNE-2 cells. The CXCR4 agent bound to CNE-2 cells (A). Single-cell signal intensity analysis showed the location of CXCR4 to be inside the cell (B). The corresponding optical dye showed a very weak signal in both the population image (C) and single-cell image (D). Statistical comparison showed that the signal intensity of CXCR4 was significantly higher than that of the free dye $(\mathrm{E})$.

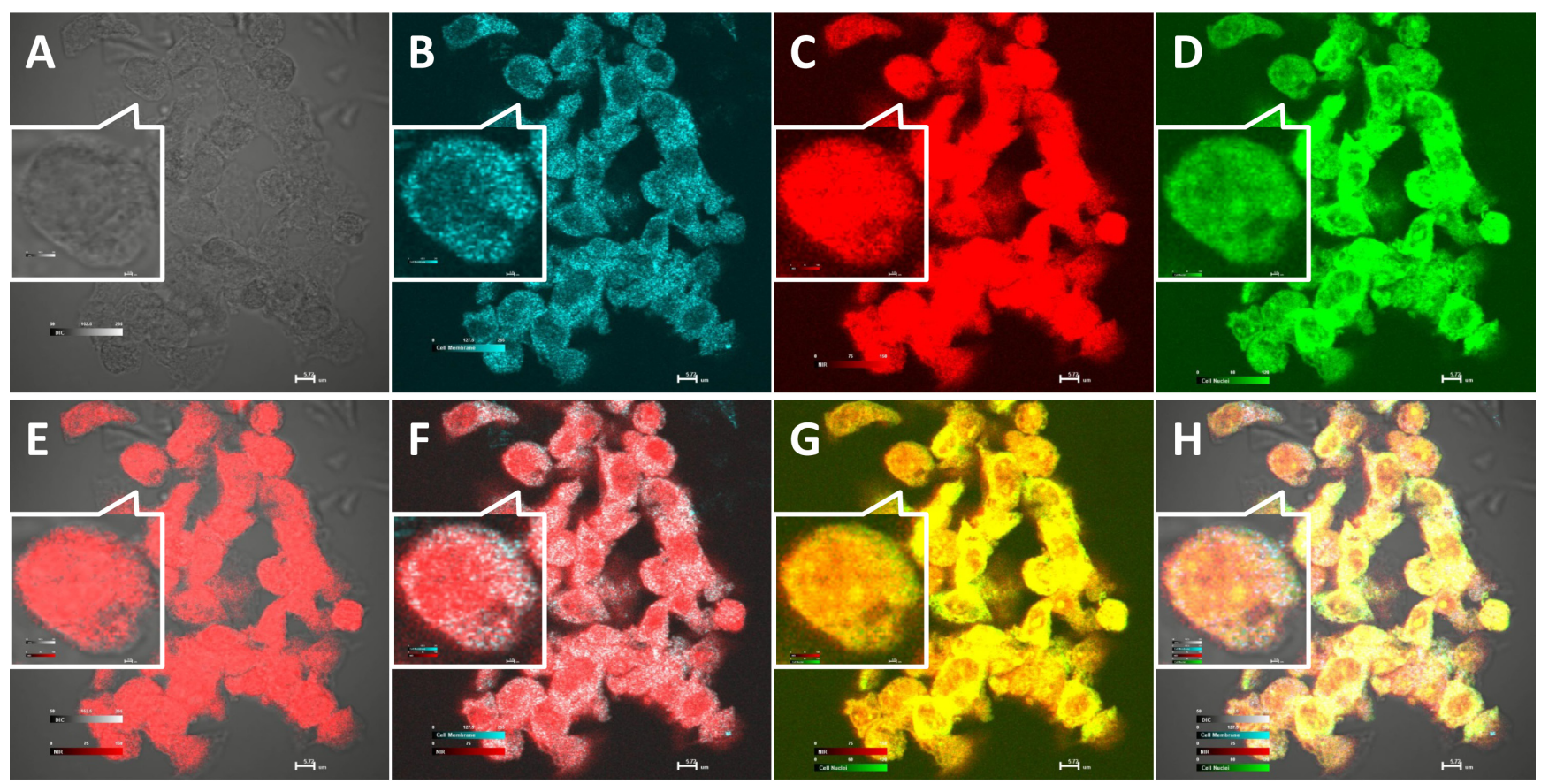

\section{Confocal images, 5-8F cell, DIC / Cell Membrane / MMP / Cell Nuclei}

Fig 3: Confocal images of human NPC 5-8F cells binding to the MMP agent. Differential interference contrast (DIC) imaging showed the cell edges and morphology (A). CellTracker ${ }^{\mathrm{TM}}$ stained the cell membranes (B). MMP bound to multiple cell components (C). Cell nuclear stain verified the cell integrity (D). Merged DIC and MMP imaging showed the MMP agent correlated to the cell morphology (E). MMP was not only present in the cell membrane (F) but also in cell nuclei (G). Merged quadruple signals showed the location of each cell compartment $(\mathrm{H})$. 


\section{In vivo imaging}

Table 1 lists injected molecular agents. Tumorbearing animals underwent common clinical imaging modalities, and the results are shown in Fig. 4. The CT body image showed the tumor location (Fig. 4A), and the CT skeleton image demonstrated that the tumor mass was a soft tissue tumor without bone damage at that stage (Fig.
4B). The ${ }^{18} \mathrm{~F}-\mathrm{FDG} / \mathrm{PET}$ image revealed that the tumor region has a higher glucose uptake than the opposite normal region (Fig. 4C). The merged PET/CT showed the ${ }^{18} \mathrm{~F}-\mathrm{FDG}$ signal intensity at the whole-body level (Fig. 4D). Analysis of the CT contrast agent intensity revealed the high vasculature structure in the tumor region (Fig. $4 \mathrm{E})$.

RA binding to the NPC tumor is shown in Fig. 5. $\mathrm{X}$-ray imaging shows the location of the tumor mass (Fig.
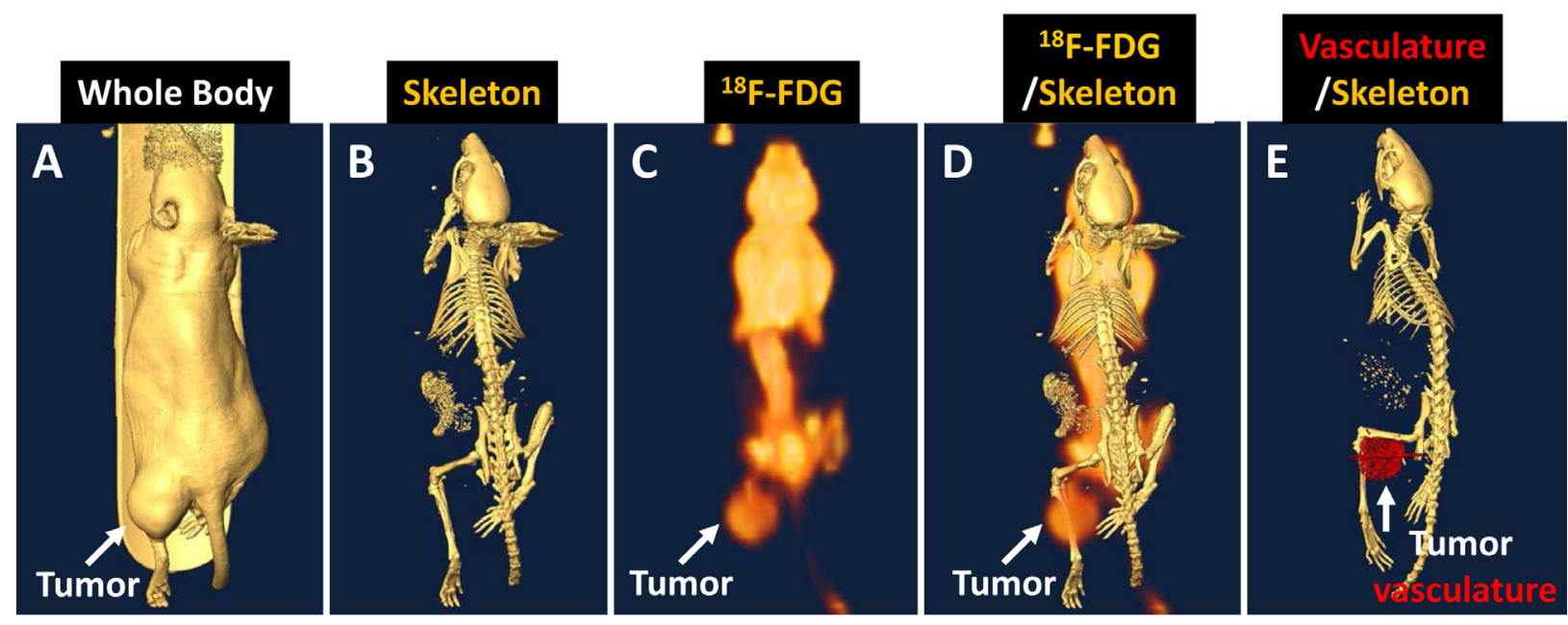

Fig 4: Human NPC tumor-bearing animal images showing the tumor location. (A), soft-tissue property without bone damage (B), glucose uptake and distribution in the whole body (C). PET/CT showed high glucose signal intensity in the tumor region (D). CT contrast agent analysis revealed the hypervasculature in the tumor region (E).
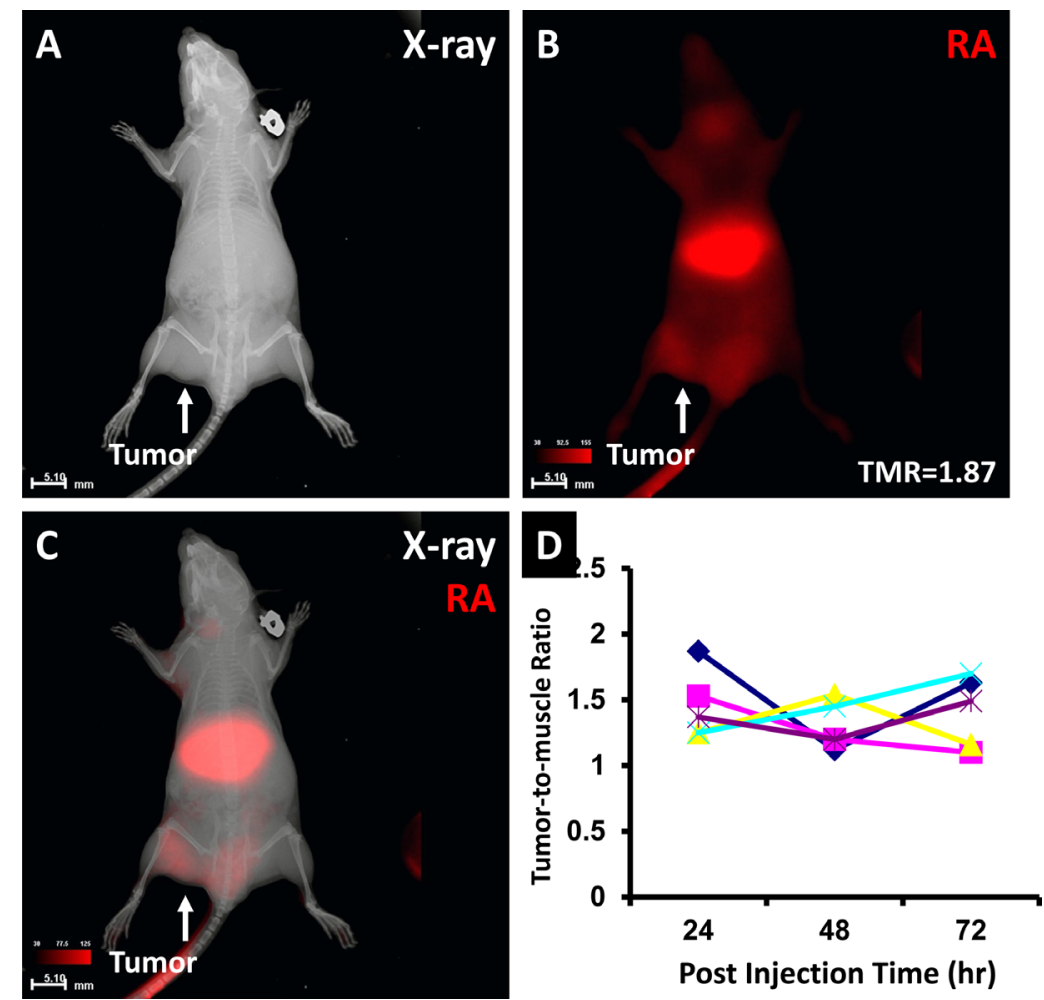

Fig 5: The RA agent binding to the human NPC xenograft. X-ray imaging showed the tumor location (A). Optical imaging showed the tumor region with a high RA signal intensity and a TMR of 1.87 (B). Merged X-ray and RA imaging showed the agent in the liver and tumor regions (C). TMR changes in each animal over 72 hour post injection demonstrated individual and dynamic variations (D). 
Table 1: Summary of molecular probes in the in vivo imaging study

\begin{tabular}{|l|l|l|l|}
\hline Target & Agent & Reporter & Injected Dose \\
\hline Glucose & 18F-FDG & $18 \mathrm{~F} 511 \mathrm{keV}$ & $100 \mu \mathrm{Ci}$ \\
\hline Vasculature & Omnipaque & lodine & $0.2 \mathrm{ml}$ \\
\hline Retinoid receptor & Retinoid acid & $\begin{array}{l}\text { IRDyeCW 780/830 nm (ex/ } \\
\text { em) }\end{array}$ & $10 \mathrm{nM}$ \\
\hline Cytokine & CXCR4 & $\begin{array}{l}\text { IRDyeCW 780/830 nm (ex/ } \\
\text { em }\end{array}$ & $5 \mathrm{nM}$ \\
\hline Stoma & $\begin{array}{l}\text { Matrix } \\
\text { metalloproteinase }\end{array}$ & $\begin{array}{l}\text { Red } \\
\text { em }\end{array}$ & $2 \mathrm{nM}$ \\
\hline
\end{tabular}

5A). Optical imaging shows high RA agent signal intensity in the tumor region with a tumor-to-muscle ratio (TMR) of 1.87. Merged X-ray and optical imaging confirmed that the signal originated from the tumor region (Fig. 5C). The TMR over post-injection time plot demonstrated the variability of each animal at different imaging times (Fig. 5D). No animal showed a consistent TMR during 72 hours.

CXCR4 binding to the NPC tumor is shown in Fig. 6. The location of the tumor xenograft is shown by X-ray imaging (Fig. 6A). The whole-body CXCR4 agent distribution is shown by optical imaging (Fig. 6B).
The tumor region has a high TMR of 1.67 at $24 \mathrm{~h}$ post injection. Merged X-ray and optical imaging demonstrated the anatomic structure and molecular imaging result (Fig. 6C). Comparison of the TMR results over 3 days post injection is shown in Fig. 6E. No statistical TMR difference was found over the 3 -day period $(\mathrm{P}=0.9884)$.

The MMP agent binding to the NPC tumor is shown in Fig. 7. Optical imaging showed high MMP signal intensity in the tumor region with a TMR of 2.56 (Fig. 7A). Merged optical and CT skeleton imaging demonstrated the nature of the soft-tissue tumor without bone destruction at this stage (Fig. 7B).
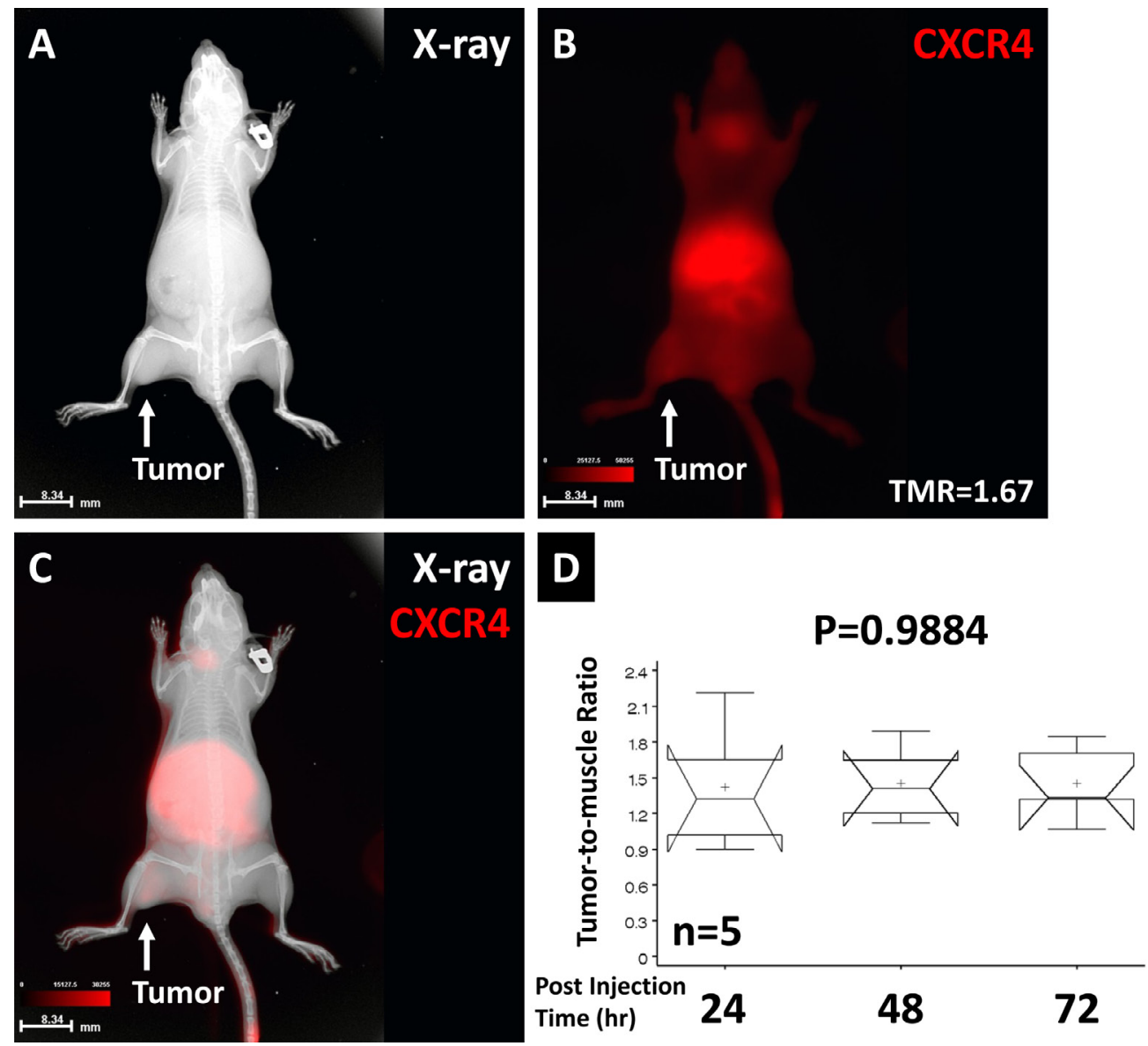

Fig 6: The CXCR4 agent binding to the NPC tumor. X-ray imaging showed the tumor location (A). The CXCR4 signal showed whole-body distribution and a high TMR in the tumor region (B). Overlaid anatomic and optical images showed the location of the CXCR4 signals (C). Comparison of TMRs over the 72-hour post injection period showed no significant difference. The high variation at the early time point suggested that the imaging agent required time to be detected in the tumor region (D). 
All imaging data were verified by dissected organ imaging (data not shown) and histopathological analysis, and the results are shown in Fig. 8. Both low- and highmagnification imaging validated the liver (Fig. 8A), muscle (Fig. 8B), and tumor (Fig. 8C).

\section{DISCUSSION}

NPC is an endemic public health problem in certain countries with an incidence rate as high as 26.9 per 100,000 persons [1-3]. It is well known that cancer is a complex, dynamic, heterogeneous systematic network of interrelations that vary in different cells, between cells, and at different times in any given cell
[7]. Therefore, developing multi-target diagnostic and therapeutic approaches becomes critical to solve this dynamic and spatially heterogeneous problem. Our previous data demonstrate that two different tumor cell lines can develop very different growth patterns in the tumor xenograft. Furthermore, the same tumor xenograft can change the growth pattern and respond to the imaging agent at different time points [14]. In addition, the same cell line in animals with the same genetic background can demonstrate different growth patterns and response to the injected agent even under near-ideal experimental conditions [19]. Together, the above results demonstrate that cancer is a complex, dynamic, heterogeneous disease and support the notion of simultaneous, multi-target diagnoses and therapies for detecting and treating cancer

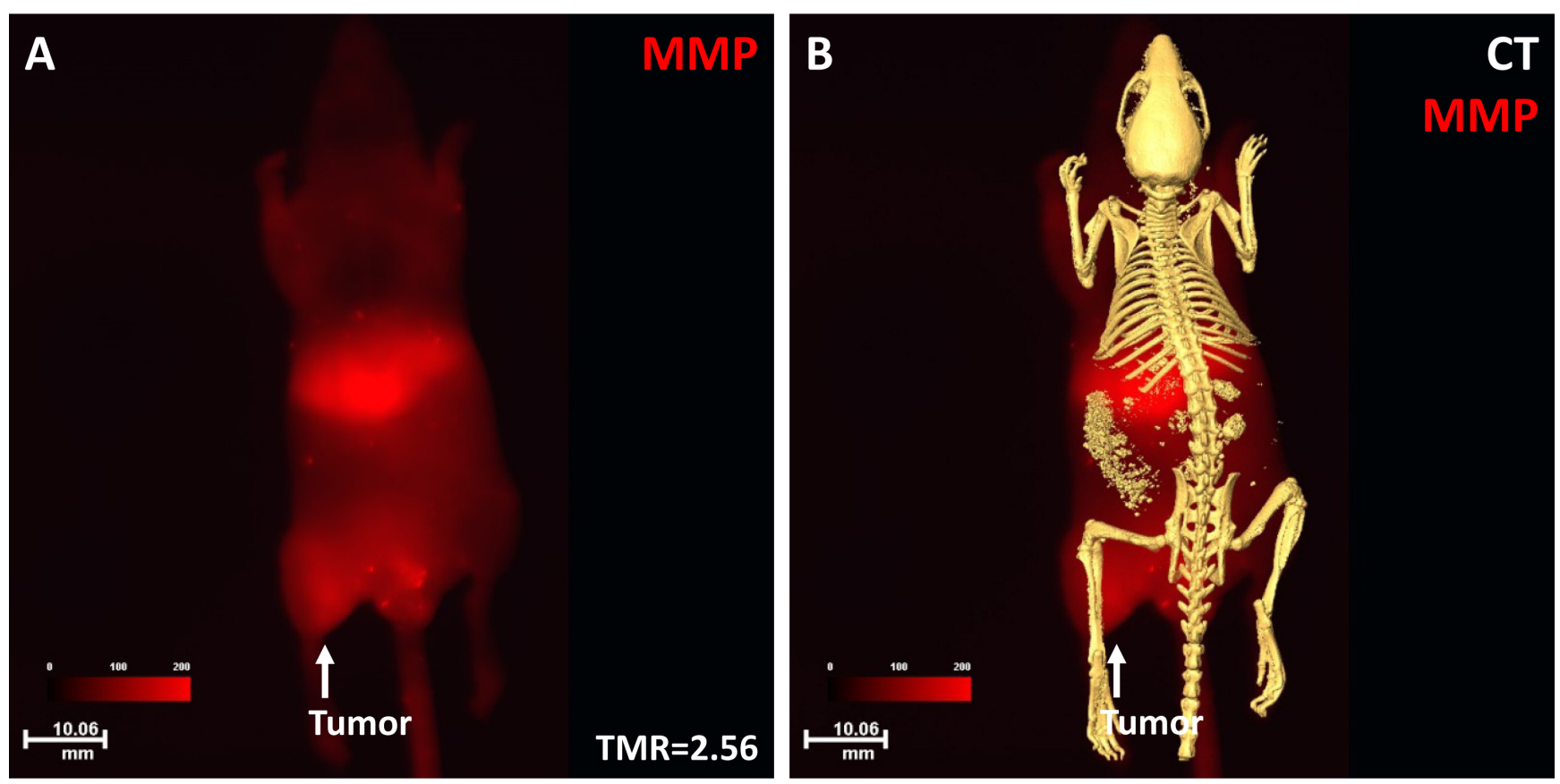

Fig 7: Optical and CT imaging of MMP binding to the NPC tumor. Optical imaging showed that the NPC tumor had high signal intensity with a TMR of 2.56 (A). The merged images showed the anatomic location of the tumor and MMP signal distribution at the whole-body level (B).
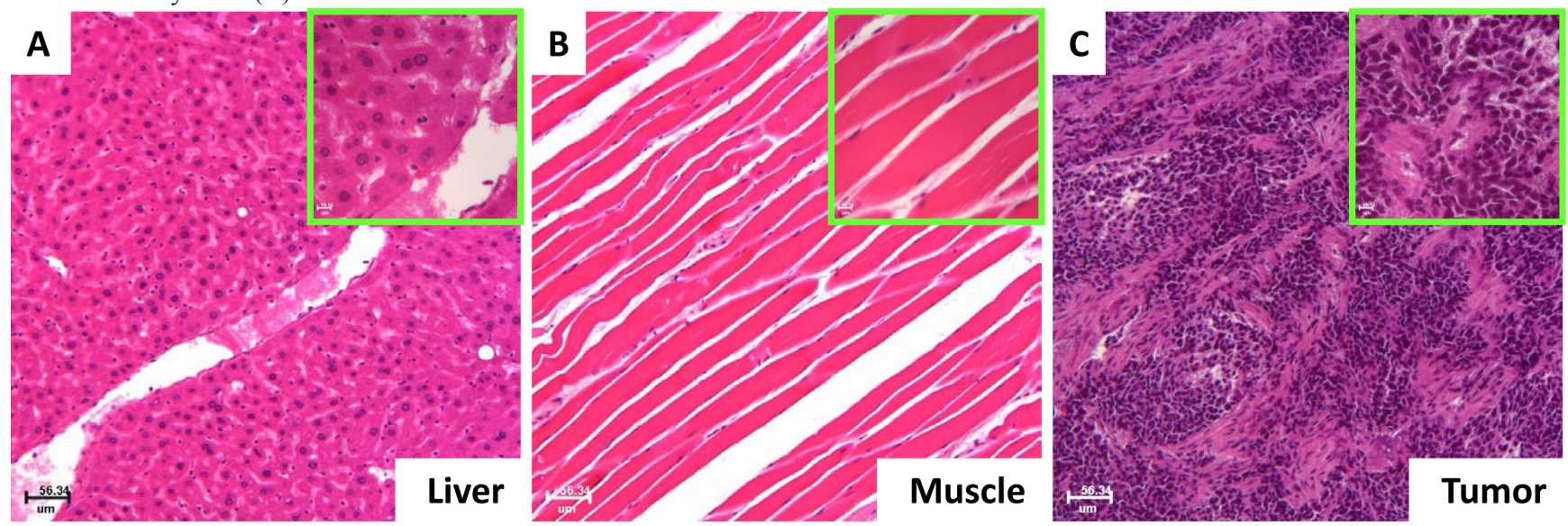

Fig 8: Histopathological validation of the imaging results. Histological results showed that the normal organ with the high signal intensity was the liver (A) and the organ with the low signal intensity was the muscle (B). The disease site with the high vasculature, high glucose, RA, CXCR4 and MMP signals represented the tumor (C). 
[7].

In the present study, we use NPC as a model to further dissect cancer heterogeneity at the cellular and molecular levels using target-specific molecular imaging. As we have demonstrated in the current study, NPC tumors can be detected by multiple agents from the cellular to whole animal levels. However, unexpectedly, our wellcharacterized RGD agent [13,14, 18-24] did not bind to any of the cell lines used in the in vitro studies. The latter result prompted us to investigate the tumor vasculature in the in vivo model using a CT contrast agent. The CT imaging data showed that NPC contains high volume of blood vessels (Fig. 4E). By contrast, our RGD agent still did not show increased signal intensity in the tumor region (data not shown). We conclude that the binding motif of the NPC tumor may be different than that of the other tumor vessels that we tested previously, and the RGD agent cannot be used as a general agent for detecting this tumor.

Given that human NPC is comprised of a mixture of multiple cells, it is logical to use multiple agents in the present study because of the complementary effects of each agent [19]. The simultaneous injection of multiple agents is expected to increase the positive detection rate. Our data demonstrated that RA, CXCR4 and MMP agents can be used both in vitro and in vivo to detect NPC. ${ }^{18} \mathrm{~F}-\mathrm{FGD} / \mathrm{PET}$ is the most commonly used molecular imaging modality in current clinical practice. However, our imaging data showed its limitation in NPC detection before the tumor metastasizes to other locations. The high glucose uptake in the normal brain and neck regions results in a low TMR and makes it difficult to distinguish the tumor from normal tissue (Fig. $4 \mathrm{C}$ and D) due to the limitation of spatial resolution.

The dynamic change in each tumor is clearly shown in Fig. 5D. Despite the increased TMR during the 72-hours post injection period, each animal shows a different TMR at each imaging time. The data demonstrate that the image only represents one time point result of dynamic interaction between the tumor and imaging agent. Furthermore, one cannot make conclusions about disease status based solely on one imaging time point with one imaging modality. Although some agents can be imaged over a long period of time without statistical difference (Fig. 6D), the variances of TMR over time re-emphasize the needs for dynamic imaging to detect dynamic tumor change.

In vivo imaging techniques have several advantages in pre-clinical researches. The combination of genetic modified the cell lines with optical reporters and injectable target-specific molecular agents let us simultaneously study tumor growth, metastasis pattern and marker expressions. The high spatial resolution of optical imaging provides the advantage of imaging tumor characteristics from whole body to cellular levels [14, 25].

Finally, the results concerning our agents that penetrate into cells suggest that they can be used as carriers for therapeutic purposes. A chemotherapeutic agent can be attached to a targeting component, changing the molecule into a target-specific chemotherapeutic agent. Because the original chemistry design is already available for this purpose, we can easily obtain three target-specific agents for treatment according to tumor marker expression status. The drawbacks of this strategy are the tissue distribution of the agent and potential organ-specific toxicity. As images have already demonstrated that all the agents show high uptake in the liver, it is obvious that the liver toxicity will more likely occur when using any of these agents at a high dose. Therefore, balancing the therapeutic efficacy and normal organ toxicity relies on capturing the therapeutic window at the correct dynamic time point for the tumor $[14,16,18,19]$. We believe noninvasive, target-specific molecular imaging will be a tool to help further understand the dynamic changes and heterogeneity in tumors.

\section{MATERIALS AND METHODS}

\section{Cell lines}

Human cancer cell lines (CNE-2, SUNE-1, 5-8F, and CCL-30) were grown in culture in Dulbecco's Modified Eagle's Medium with high glucose or F12 medium (Life Technologies, Grand Island, NY), supplemented with 10\% fetal bovine serum (Hyclone, Logan, UT), in incubators with $5 \% \mathrm{CO}_{2}$ at $37^{\circ} \mathrm{C}$.

\section{Tumor xenografts}

Nude mice (4- to 6-week-old; 18-22 g; Harlan, Indianapolis, IN) were maintained in a pathogen-free mouse colony in a facility accredited by the American Association for Laboratory Animal Care, and all experiments were performed in compliance with the guidelines of the Institutional Animal Care and Use Committee. For tumor implantation, cultured tumor cells were harvested near confluence by treating monolayers with $0.05 \%$ trypsin-ethylenedinitrilotetraacetic acid. Cells were pelleted at $130 \times g$ for $5 \mathrm{~min}$ and resuspended in sterile phosphate-buffered saline. Approximately $1 \times 10^{6}$ cells were implanted subcutaneously into each mouse.

\section{Imaging agent synthesis}

All agents were designed and synthesized in-house as previously described [8-19]. They were purified by high-performance liquid chromatography (HPLC) and confirmed by mass spectrometry, analytic HPLC, and fluorescence spectrophotometry. ${ }^{18} \mathrm{~F}-\mathrm{FDG}$ was purchased from Cyclotope (Houston, TX). The CT contrast agent 
Omnipaque was purchased from GE Healthcare (Fairfield, CT).

\section{Confocal microscopic imaging}

The human NPC cell lines 5-8F, CCL-30, CNE-2, and SUNE-1 were used for in vitro binding analysis. All cells were co-incubated with either the imaging agents or their reporter dye for $30 \mathrm{~min}$ at $37^{\circ} \mathrm{C}$. Cells were fixed and counterstained with 1 microM Sytox Green (Life Technologies) in $95 \%$ ethanol for $15 \mathrm{~min}$ at $4^{\circ} \mathrm{C}$. For cell membrane staining, cells were incubated with membrane dye (CellTracker ${ }^{\mathrm{TM}}$; Life Technologies) for $10 \mathrm{~min}$ at $37^{\circ} \mathrm{C}$, and then for $20 \mathrm{~min}$ at $4^{\circ} \mathrm{C}$.

Stained cells were transferred to slides for microscopic examination. Images were captured using an Olympus confocal microscope (Fluoview 1000; Olympus America, Center Valley, PA). Near-infrared (NIR) dyes were measured at excitation/emission (Ex/ Em) wavelengths of $765 / 810 \mathrm{~nm}$, the cell membrane was examined at 553/570, and cell nuclei were examined at $488 / 510 \mathrm{~nm}$. Signal intensities were recorded from one slice of multiple z-stacks with 0.5-micrometer gaps. Sytox Green, membrane imaging agent or NIR dye signals were pseudocolor green (Em $510 \mathrm{~nm})$, Cyan (Em $570 \mathrm{~nm}$ ) or red $(\mathrm{Em} 810 \mathrm{~nm})$, respectively.

\section{Animal imaging}

Tumors developed after 3 to 4 weeks of growth in the implanted mice to $8-15 \mathrm{~mm}$ in diameter. Imaging agents $(2-10 \mathrm{nM})$ were injected into the tail vein of anesthetized mice. Mice were imaged immediately after injection and for as long as 72 hours subsequently. Optical and X-ray images were recorded using the Bruker InVivo Multispectral System FX Pro instrument (Bruker Preclinical Imaging, Billerica, MA). In vivo PET/singlephoton emission CT (SPECT)/CT imaging was performed using the Siemens MicroCAT II SPECT/CT and Inveon PET instrument (Siemens Medical Solutions, Malvern, PA).

\section{Statistical analysis}

SAS software v9.3 (SAS Institute, Cary, NC) was used to analyze data by one-way analysis of variance or the general linear model. Data comparison was presented in notched box-and-whisker plots. The medians (central lines) of two box-and-whisker plots were considered to be significantly different at the 0.05 level ( $95 \%$ confidence), if the corresponding notches did not overlap.

\section{CONCLUSION}

We report preclinical research regarding the use of multi-target-specific molecular imaging agents to determine NPC heterogeneity disease components at the cellular level. More importantly, our data support cancer as a complex network of interrelations that vary in different cells, between cells, and at different times in any given cell. We should consider cancer as an evolving, dynamic, and heterogeneous system and treat cancer using simultaneous, multi-target therapies [7].

\section{ABBREVIATIONS}

$\begin{array}{ll}\mathrm{CT} & =\text { Computed tomography } \\ \mathrm{DIC} & =\text { Differential interference contrast } \\ \mathrm{Em} & =\text { Emission } \\ \mathrm{Ex}=\text { Excitation } & \\ \text { FDG or }{ }^{18} \text { F-FDG = Fluorodeoxyglucose } \\ \text { HPLC } \quad=\text { High-performance liquid } \\ \text { chromatography } \\ \begin{array}{ll}\mathrm{MMP} & =\text { Matrix metalloproteinase } \\ \mathrm{NPC} & =\text { Nasopharyngeal carcinoma } \\ \mathrm{PBS} & =\text { Phosphate-buffered saline } \\ \mathrm{PET} & =\text { Positron emission tomography } \\ \mathrm{RA} & =\text { Retinoid acid } \\ \text { TMR } & =\text { Tumor-to-muscle ratio }\end{array}\end{array}$

\section{CONFLICT OF INTEREST}

The authors declare there are no financial or nonfinancial competing interests.

\section{ACKNOWLEDGMENTS}

Grant support was provided by the National Natural Science Foundation of China 81071797 (F.Z.), and Guangdong Scientific Bureau Research Fund 2009B080701071 (F.Z.); Guangdong Education Research Fund 2012B091100458 (F.Z.); Guangzhou Scientific Research Fund 20130000013 (F.Z.); the Department of Defense grant W81XWH-08-1-0489 (S.K., W.W.).

We thank Dr. Zhi-Dong Jiang for critically reviewing the manuscript and providing suggestions. We also thank BioScience Writers, LLC, Houston, TX, for providing scientific editing services.

\section{REFERENCES}

1. Ohno T, Thinh DH, Kato S, Devi CR, Tung NT, Thephamongkhol K, Calaguas MJ, Zhou J, Chansilpa Y, Supriana N, Erawati D, Banu PA, Koo CC, Kobayashi K, Nakano T and Tsujii H. Radiotherapy concurrently with weekly cisplatin, followed by adjuvant chemotherapy, 
for N2-3 nasopharyngeal cancer: a multicenter trial of the Forum for Nuclear Cooperation in Asia. Journal of radiation research. 2012.

2. Yu MC and Yuan JM. Epidemiology of nasopharyngeal carcinoma. Seminars in cancer biology. 2002; 12(6):421429.

3. Chan AT and Felip E. Nasopharyngeal cancer: ESMO clinical recommendations for diagnosis, treatment and follow-up. Ann Oncol. 2009; 20 Suppl 4:123-125.

4. Li YZ, Cai PQ, Xie CM, Huang ZL, Zhang GY, Wu YP, Liu LZ, Lu CY, Zhong R and Wu PH. Nasopharyngeal cancer: impact of skull base invasion on patients prognosis and its potential implications on TNM staging. European journal of radiology. 2013; 82(3):e107-111.

5. Su CY and Lui CC. Perineural invasion of the trigeminal nerve in patients with nasopharyngeal carcinoma. Imaging and clinical correlations. Cancer. 1996; 78(10):2063-2069.

6. Chan AT, Gregoire V, Lefebvre JL, Licitra L, Hui EP, Leung SF, Felip E and Group E-E-EGW. Nasopharyngeal cancer: EHNS-ESMO-ESTRO Clinical Practice Guidelines for diagnosis, treatment and follow-up. Ann Oncol. 2012; 23 Suppl 7:vii83-85.

7. Floor SL, Dumont JE, Maenhaut C and Raspe E. Hallmarks of cancer: of all cancer cells, all the time? Trends in Molecular Medicine. 2012; 18(9):509-515.

8. Wang W, Ke S, Kwon S, Yallampalli S, Cameron AG, Adams KE, Mawad ME and Sevick-Muraca EM. A new optical and nuclear dual-labeled imaging agent targeting interleukin 11 receptor alpha-chain. Bioconjug Chem. 2007; 18(2):397-402.

9. Wang W, Ke S, Wang X, Charnsangavej C, Gelovani JG and Li C. Synthesis and evaluation of a new near-iInfrared fluorescence (NIRF) imaging agent targeted to matrix metalloproteinase-2. . Molecular Imaging 2005; 4(3):282.

10. Auld DS, Lovell S, Thorne N, Lea WA, Maloney DJ, Shen M, Rai G, Battaile KP, Thomas CJ, Simeonov A, Hanzlik RP and Inglese J. Molecular basis for the high-affinity binding and stabilization of firefly luciferase by PTC124. Proc Natl Acad Sci U S A. 107(11):4878-4883.

11. Sampath L, Kwon S, Ke S, Wang W, Schiff R, Mawad ME and Sevick-Muraca EM. Dual-labeled trastuzumab-based imaging agent for the detection of human epidermal growth factor receptor 2 overexpression in breast cancer. J Nucl Med. 2007; 48(9):1501-1510.

12. Wang W, Shao R, Wu Q, Ke S, McMurray J, Lang FF, Jr., Charnsangavej C, Gelovani JG and Li C. Targeting gelatinases with a near-infrared fluorescent cyclic His-TryGly-Phe peptide. Mol Imaging Biol. 2009; 11(6):424-433.

13. Wang W, Ke S, Wu Q, Charnsangavej C, Gurfinkel M, Gelovani JG, Abbruzzese JL, Sevick-Muraca EM and Li C. Near-infrared optical imaging of integrin alphavbeta3 in human tumor xenografts. Mol Imaging. 2004; 3(4):343-351.

14. Wang W, Cameron AG, Wendt JA, Mawad ME and Ke S. Multi-wavelength Optical Imaging of Human Tumor
Xenografts. Australian Journal of Chemistry. 2011; 64(5):625-632.

15. Wang W, Lin J, Guha S, Tong Z, Cameron AG, Zhang F, Qiu X, Zou C, Gao X, Mawad ME and Ke S. TargetSpecific Agents Imaging Ectopic and Orthotopic Human Pancreatic Cancer Xenografts. Pancreas. 2011; 40(5):689694.

16. Wang W, Qiu X, Zhang F, Sun J, Cameron AG, Wendt JA, Mawad ME and Ke S. An imageable retinoid acid derivative to detect human cancer xenografts and study therapeutic dosing to reduce its toxicity. Contrast Media Mol Imaging. 2011; 6:200-208.

17. Ke S, Wang W, Qiu X, Zhang F, Anderson ML, Mach C, Konopleva M, Yustein JT, Zou C, Gao X, Lin J, Sun J and Cameron AG. (2012). Retinoic Acid: An Old Compound with a Potential for Future Use in Personalized Molecular Medicine. (Hauppauge: Nova Science Publishers ).

18. Ke S, Zhang F, Wang W, Qiu X, Lin J, Cameron AG, Zou C, Gao X, Zou C, Zhu VF and Li M. Multiple target-specific molecular imaging agents detect liver cancer in a preclinical model. Current molecular medicine. 2012; 12(8):944-951.

19. Ke S, Wang W, Qiu X, Zhang F, Yustein JT, Cameron AG, Zhang S, Yu D, Zou C, Gao X, Lin J, Yallampalli S and Li M. Multiple target-specific molecular agents for detection and image analysis of breast cancer characteristics in mice. Current molecular medicine. 2013; 13(3):446-458.

20. Gurfinkel M, Ke S, Wang W, Li C and Sevick-Muraca EM. Quantifying molecular specificity of alphavbeta3 integrintargeted optical contrast agents with dynamic optical imaging. J Biomed Opt. 2005; 10(3):034019.

21. Houston JP, Ke S, Wang W, Li C and Sevick-Muraca EM. Quality analysis of in vivo near-infrared fluorescence and conventional gamma images acquired using a dual-labeled tumor-targeting probe. J Biomed Opt. 2005; 10(5):054010.

22. Kwon S, Ke S, Gurfinkel M, Wang W, Wu QP, Li C and Sevick-Muraca EM. Dose-dependent uptakes of an RGDfluorescent dye-targeted to receptor expressed Kaposi's Sarcoma. Molecular Imaging. 2005; 4(2):1-14.

23. Kwon S, Ke S, Houston JP, Wang W, Wu Q, Li C and Sevick-Muraca EM. Imaging dose-dependent pharmacokinetics of an RGD-fluorescent dye conjugate targeted to alpha v beta 3 receptor expressed in Kaposi's sarcoma. Mol Imaging. 2005; 4(2):75-87.

24. Li C, Wang W, Wu Q, Ke S, Houston J, Sevick-Muraca E, Dong L, Chow D, Charnsangavej C and Gelovani JG. Dual optical and nuclear imaging in human melanoma xenografts using a single targeted imaging probe. Nucl Med Biol. 2006; 33(3):349-358.

25. Vigdis Nygaard LP, Kotryna Vasiliauskaite, Trevor Clancy and Eivind Hovig. Melanoma brain colonization involves the emergence of a brain-adaptive phenotype. Oncoscience. 2014; 1(1):13. 